\title{
Estudos florísticos no município de Presidente Figueiredo, Amazonas, Brasil - Il: famílias Myristicaceae, Siparunaceae e Monimiaceae
}

\author{
José Augusto Coelho da SILVA ${ }^{1}$, Marlene Freitas da SILVA',3
}

\section{RESUMO}

O Município de Presidente Figueiredo, do Estado do Amazonas, Brasil, foi visitado durante três anos consecutivos (1996 a 1999), com o objetivo de obter-se amostras botânicas da área para estudos do Subprojeto "Elaboração de Revisões e Monografias Taxonômicas", com a finalidade de elaborar-se a Flórula para o Município. As coleçôes feitas nesse período encontram-se depositadas no acervo do Herbário do INPA e as informaçôes gerais sobre os taxa aqui estudados foram complementadas com as obtidas das coleções feitas anteriormente por outros pesquisadores. Para a família Myristicaceae Br. R. foram identificadas 23 espécies e duas variedades, distribuídas em quatro gêneros. Destes, Iryanthera Warb. e Virola Aubl. destacaram-se pela diversidade em espécies, sendo Iryanthera ulei Warb. e Virola calophylla (Spruce) Warb. var. calophylla as espécies mais coletadas. A família Monimiaceae Juss. está representada apenas pelo gênero Mollinedia Ruiz \& Pavón, pela espécie M. ovata Ruiz \& Pavón; já a família Siparunaceae (A. DC.) Schodde encontra-se representada pelo gênero Siparuna Aubl. com oito espécies, sendo S. cristata (Poepp. \& Endl.) A. DC. a espécie mais coletada.

PALAVRAS-CHAVE: Presidente Figueiredo, Lista de espécies, Myristicaceae, Monimiaceae, Siparunaceae.

\section{Floristic studies in township of Presidente Figueiredo, Amazonas, Brazil - II: families Myristicaceae, Siparunaceae and Monimiaceae}

\section{ABSTRACT}

The Township of Presidente Figueiredo, in the State of Amazonas, Brazil, was visited for three consecutive years, from 1996 to 1999 , with the aim of acquiring botanical samples from the area, in order to carry out studies on the "Taxonomic Monographies and Revisions Preparation" Subproject for the purpose of preparing a survey of the flora. Collections pertaining to the present work are deposited at INPA's herbarium and, general information on the taxa studied here were complemented with those obtained from earlier collections conducted by other researchers. Twenty three (23) species and two varieties, distributed in four genera were identified for family Myristicaceae Br. R. Of these Iryanthera Warb. and Virola Aubl. stood out on account of their species diversity, being Iryanthera ulei Warb. and Virola calophylla (Spruce) Warb. var. calophylla the most collected ones. Family Monimiaceae Juss. is only represented by genus Mollinedia Ruiz \& Pavón through species $M$. ovata Ruiz \& Pavón, yet family Siparunaceae (A. DC.) Schodde is represented by genus Siparuna Aubl. with eight species, being S. cristata (Poepp. \& Endl.) A. DC. the most collected one.

KEYWORDS: Presidente Figueiredo, Check list, Myristicaceae, Monimiaceae, Siparunaceae.

1 Instituto Nacional de Pesquisas da Amazônia (INPA), Coordenação de Pesquisas em Botânica, Caixa Postal 478, CEP 69.060-001 - Manaus AM. e-mail: augusto@inpa.gov.br 2 Universidade do Estado do Amazonas (UEA), Coordenadora do Curso de Pós-Graduação em Biotecnologia de Produtos Naturais da Amazônia e Professora Titular do Curso de Engenharia Florestal da UEA. Instituto de Tecnologia da Amazônia (UTAM), Av. Darcy Vargas, 1200, CEP 69050-020.

3 In memorian 


\section{INTRODUÇÃO}

O Município de Presidente Figueiredo está situado a nordeste do Estado do Amazonas, norte de Manaus, e é delimitado pela linha do Equador, paralelo $3^{\circ} 00^{\prime} 00^{\prime \prime}$ e pelos meridianos 61030'00" e 58³0'00", perfazendo uma área de $24.781 \mathrm{~km}^{2}$ (Figura 1).

A cobertura vegetal do Município é constituída, principalmente, por floresta ombrófila densa de terra firme e os principais acidentes geográficos são cachoeiras, corredeiras, cavernas, igarapés, lagos e rios que, devido às inundaçōes periódicas, durante a estação chuvosa, mantêm em suas margens a floresta de igapó.

Embora a região tenha sofrido uma grande exploração seletiva do pau-rosa (Aniba rosaeodora Ducke, Lauraceae), a vegetação do Município é ainda em grande parte constituída por florestas naturais, com poucas áreas perturbadas pela ação antrópica (Eletronorte/IBAMA, 1996).

A família Myristicaceae é amplamente distribuída pelas regiōes tropicais e subtropicais do Novo e Velho Mundo. Segundo Rodrigues (1980), o centro de distribuição mundial dessa família pantropical é a Ásia tropical, da Índia à Polinésia. Além da América e Ásia, a família está também representada na África e em Madagascar. Na América ela ocorre nos dois hemisférios, desde o sul do México e Antilhas Menores até Bolívia e parte setentrional do Rio Grande do Sul, no Brasil (Belota Filho, 1994). Os gêneros formam grupos geograficamente bem definidos e nenhum dos gêneros desses grupos ocorre naturalmente, em qualquer outra dessas quatro áreas maiores de especiação.

Caracterizada principalmente, por apresentar quase que exclusivamente árvores medianas, arvoretas e árvores de dossel, raramente arbustos, a família Myristicaceae pode ser reconhecida, quando em estado vegetativo, por algumas características peculiares, como: tronco com entrenós bem definidos; casca do tronco, quando cortada ou ferida, exsudando seiva avermelhada em geral abundante e translúcida que em contato com o ar se oxida, passando a vermelho (exceto em Osteophloeum Warb.); folhas coriáceas, inteiras e sem estípulas; pecíolo profundamente canaliculado; filotaxia alterna, dística (Ribeiro et al., 1999).

Constituída por cerca de 18 gêneros e 400 espécies as Miristicáceas nas Américas são representadas por cinco gêneros endêmicos, com aproximadamente 93 espécies: Virola Aublet (45 spp.), Iryanthera Warb. (24 spp.), Compsoneura Warb. (16 spp.), Otoba A. DC. ex Karsten. (7 spp.) e Osteophloeum Warb. (1 sp.). Família de distribuição considerável em algumas regiōes constitui-se num dos mais importantes elementos da flora regional. Particularmente, segundo Belota Filho (1994), a bacia amazônica é o centro de distribuição da família no continente sul-americano, onde certas espécies são muito comuns.
Muitas espécies do gênero Virola são de grande importância ecológica e econômica sendo usadas em larga escala na fabricação de caixas, móveis, tanoaria, compensados, laminados e contraplacados (Loureiro et al., 1989). E, como consequiência do processo industrial, permite o uso dos resíduos de produção na confecção de papel tipo Kraft de boa qualidade, além de ser uma boa madeira para o aproveitamento racional, como fonte de celulose e papel.

Rodrigues (1980) destacou ainda que algumas espécies desse gênero são usadas na medicina popular para a cura do reumatismo, artritismo em geral, cólicas, dispepsias e erisipelas; o cozimento da casca destas espécies é utilizado na assepsia de feridas, como coadjuvante na cicatrização (Le Cointe, 1947).

As famílias Monimiaceae e Siparunaceae foram recentemente segregadas: a primeira, é considerada muito heterogênea; estudos mais recentes apontam para a segregação de certos gêneros como Siparuna Aubl., para constituir a família Siparunaceae (Schodde, 1970; Ribeiro et al., 1999). Essas famílias, por serem vegetativamente semelhantes, serão aqui tratadas conjuntamente.

De acordo com Ribeiro et al. (1999), as Siparunáceas são neotropicais com dois gêneros: Glossocalyx Benth. e Siparuna Aubl. O primeiro, Glossocalyx é um gênero mono-específico na África Ocidental, porém, com cerca de 70 espécies distribuídas desde o México e o Caribe, até o Paraguai e Argentina. Na Amazônia brasileira está representada pelo gênero Siparuna Aubl.

Em sua forma vegetativa de vida a família pode ser facilmente reconhecida pelo hábito arbóreo ou arbustivo, bem como pela combinação das seguintes características: odor cítrico desagradável ou fétido de toda a planta (daí o nome indígena caá-pitiú = planta de cheiro ruim), devido à grande concentração de óleos essenciais; folhas geralmente simples, elípticas (exceto em S. monogyna em que a folha é obovada), decussadas, verticiladas ou opostas, de margens inteiras a denteadas ou serreadas, sem estípulas; pecíolo de comprimento variável; ramos cilíndricos com nós achatados; algumas espécies são completamente glabras enquanto outras apresentam vários tipos de indumento de pêlos nas folhas ou nos ramos; pêlos simples, estrelados ou escamosos (Nee, 1995; Ribeiro et al., 1999).

A família Monimiaceae com 22 gêneros e, aproximadamente, 194 espécies é predominantemente pantropical e apresenta a mesma distribuição das Siparunáceas (Ribeiro et al., 1999). Na Amazônia brasileira, a família está representada pelos gêneros Bracteanthus Ducke e Mollinedia Ruiz \& Pavón.

Em seu habitat natural a família Monimiaceae é reconhecida pelas mesmas características vegetativas da família Siparunaceae. Siparuna cuspidata é utilizada no tratamento 
de disenteria e, a madeira de algumas espécies do gênero é utilizada na construção civil, na obtenção de essências e na medicina popular (Nee, 1995; Ribeiro et al., 1999).

No Equador, Mollinedia ovata é usada pelos indígenas para tratar febres, dores de cabeça ou problemas de estômago.

\section{ÁREA DE ESTUDO}

Presidente Figueiredo é um dos municípios amazonenses de melhor e maior acesso aos visitantes de todo o estado e que, pela exuberância de seus recursos naturais, apresenta grande potencial para o turismo ecológico, por incorporar em seus limites, o lago da Hidrelétrica de Balbina, propício à prática da pesca desportiva, além de outras atividades turísticas como, espeleologia, a pesquisa arqueológica, entre outras.

Entretanto, o grande número de visitantes e a carência de um programa de educação ambiental para a preservação das espécies locais trouxeram a preocupação pelo possível desaparecimento de algumas espécies, daí a proposta de elaborar-se um estudo florístico para o Município e assim contribuir com o turismo ecológico, identificando as espécies vegetais ali existentes, bem como a área de distribuição dessas espécies, por meio do levantamento das coleções depositadas no acervo do Herbário do INPA.

Fatores naturais como clima, relevo, tempo e materiais de origem combinados em intensidades diferentes, formam os diversos tipos de solo observados na região. Duas unidades destacam-se entre esses tipos de solo: os podzólicos vermelho álico e/ou distrófico e, os latossolos vermelho amarelo álico e/ou distrófico. Ocorrem ainda; areias quartzosas, laterita hidromórfica e latossolo amarelo (Nava et al., 1998).

O clima da região, segundo a classificação de Köppen (1948) é do tipo Amw caracterizado por apresentar-se chuvoso, úmido e quente, com maior incidência de chuvas no período de dezembro a maio. Regionalmente, a temperatura é uniforme ao longo de todo o ano, variando entre a máxima

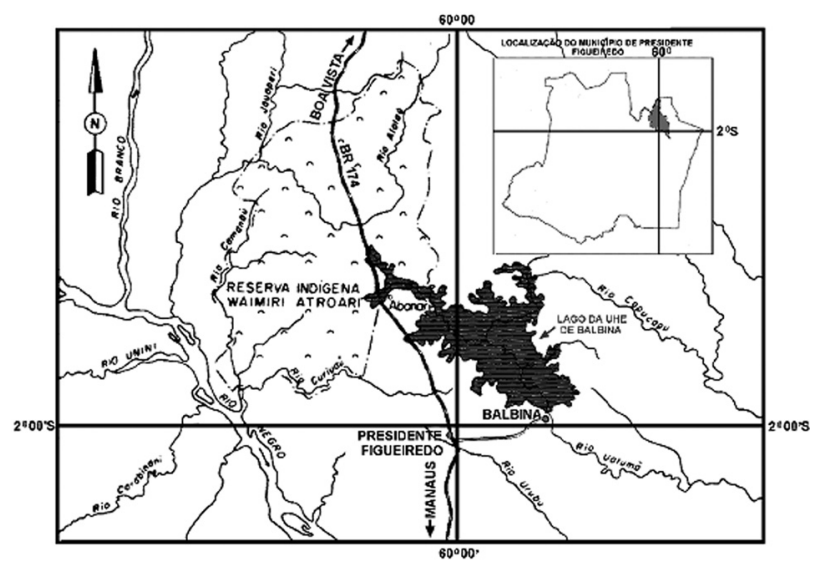

Figura 1 - Mapa de localização do Município de Presidente Figueiredo, AM, Brasil, Escala aproximada 1:2. 000.000. (Nava et al.,1998). de $38^{\circ} \mathrm{C}$ e a mínima de $20^{\circ} \mathrm{C}$. A umidade relativa do ar apresenta-se alta e uniforme durante o ano, sendo de $97 \%$ o valor médio (Nava et al., 1998).

\section{MATERIAL E MÉTODOS}

O material botânico para esta pesquisa foi coletado no Município de Presidente Figueiredo, AM, Brasil no período de 1996 a 1999 e as observações relativas às espécies foram complementadas com as contidas nas etiquetas dos espécimes adicionais depositados no herbário do INPA. O material fértil foi processado conforme normas clássicas para esse tipo de trabalho, obedecendo-se às recomendações do INPA com relação á incorporação das coleções básicas após identificadas ao acervo do herbário.

Para identificação das espécies usou-se o método clássico da morfologia comparada, utilizando-se espécimes herborizados, já identificados por especialistas, comparando-os com o material ainda fresco. Para a certificação dos nomes das espécies foi utilizada a bibliografia clássica especializada usada em taxonomia, tais como: Index Kewensis (Hooker \& Jackson, 1895-1974), Gray Herbarium Index (1968-1978), Flora Brasiliensis de Martius (De Candolle, 1860 e Tulasne, 1857), Taxonomic Literature (Stafleu, 1967) e o Código Internacional de Nomenclatura de Botânica (Greuter et al. 2003).

\section{RESULTADOS}

Do material coletado foram identificadas 23 espécies e duas variedades da família Myristicaceae distribuídas em quatro gêneros: Virola Aubl., Iryanthera Warb., Compsoneura Warb., Otoba A. DC. ex Karsten. e Osteophloeum Warb. Para Siparunaceae foram identificadas oito espécies do gênero Siparuna Aubl. e, para a família Monimiaceae uma espécie, todas relacionadas na Tabela 1 .

\section{DISCUSSÃO}

Dentre as três famílias estudadas, para o Município de Presidente Figueiredo, as que apresentaram maior diversidade de gêneros e espécies, foram: Myristicaceae com quatro gêneros, 23 espécies e duas variedades; Siparunaceae com seu único gênero representado na Amazônia brasileira, com oito espécies.

Os gêneros da família Myristicaceae com maior número de espécies foram Iryanthera Warb. com 11 espécies e, Virola Aubl. com 10 espécies e duas variedades. 
Iryanthera ulei Warb. foi a espécie coletada mais vezes, tendo sido coletada 17 vezes, seguida de Virola calophylla (Spruce) Warb. var. calophylla coletada 14 vezes, Compsoneura ulei Warb., Virola multinervia Ducke e Virola venosa (Benth.) Warb. coletadas seis vezes, Iryanthera laevis Mgf., Iryanthera lancifolia Ducke, Osteophloeum platyspermum (Spruce ex A. DC.) Warb., Virola elongata (Benth.) Warb., Virola mollissima (Poepp. ex A. DC.) Warb. e Virola pavonis (A. DC.) A. C. Smith coletadas cinco vezes.

Para Siparunaceae, família monotípica, o gênero Siparuna Aubl. está representado por oito espécies, sendo a mais abundante Siparuna cristata (Poepp. \& Endl.) A. DC coletada seis vezes.

Tiveram menor representatividade as demais espécies relacionadas na Tabela 1, as quais foram coletadas, apenas, entre uma e quatro vezes, respectivamente.

Estas famílias encontradas são comuns a outros municípios da regiāo. Para a Flora da Reserva Ducke próximo ao Município de Manaus, Ribeiro et al. (1999) citou a maioria dos gêneros relacionados para Presidente Figueiredo. Nee (1995), também relacionou em sua Flora Preliminar, as famílias e gêneros encontrados em Presidente Figueiredo.

Com relação aos espécimes identificados apenas no nível genérico, não foi possível estabelecer afinidades estreitas com as espécies já identificadas no acervo do Herbário do INPA, indicando, possivelmente, serem espécies novas para a ciência ou novos registros para a região amazônica. Contudo, estas dúvidas somente poderão ser esclarecidas, procedendo-se a um estudo mais aprofundado, pelos especialistas dessas famílias.

Convém ressaltar que embora representativos, os dados aqui encontrados não devem ser considerados como absolutos, mesmo porque o total das espécies aqui relacionadas pode não corresponder, eventualmente, ao número total real para as famílias identificadas para Presidente Figueiredo. Novas coletas, certamente, levarão a um aumento no número de espécies e, a determinação da diversidade real dessas famílias só estará completa quando, por meio de sucessivas coletas, nos diferentes ecossistemas da região, não forem encontradas novas entidades.

Tabela 1 - Famílias e Espécies encontradas, Nome e Números dos coletores e Freqüência de coleta, no Município de Presidente Figueiredo(AM): Myristicaceae, Siparunaceae e Monimiaceae.

\begin{tabular}{|c|c|c|}
\hline Famílias/Espécies & $\begin{array}{l}N^{0} \text { de } \\
\text { Ocorrência }\end{array}$ & Nome e $\mathrm{N}^{0}$ de Coletores \\
\hline \multicolumn{3}{|l|}{ Myristicaceae } \\
\hline Compsoneura ulei Warb. & 6 & $\begin{array}{l}\text { Bisby, F. et al. P18097; Loureiro, A. et al. s.n. (INPA 47928), s.n. (INPA 48055), s.n. (INPA } \\
\text { 48150), s.n. (INPA 48204); Thomas, W. et al. } 5401 .\end{array}$ \\
\hline Iryanthera coriacea Ducke & 4 & $\begin{array}{l}\text { Berg, C. C. et al. P19469; Loureiro, A. et al. s.n. (INPA 47924), s.n. (INPA 48104), s.n. } \\
\text { (INPA 48156). }\end{array}$ \\
\hline Iryanthera juruensis Warb. & 4 & Loureiro A. et al. s.n. (INPA 47895), s.n. (INPA 48197); Thomas, W. et al. 5242, 5286. \\
\hline Iryanthera laevis Mgf. & 5 & $\begin{array}{l}\text { Bisby, F. et al. P18088; Cid, C. A. et al. 6958; Loureiro, A. et al. s.n. (INPA 43846); } \\
\text { Rodrigues, W. A. et al. s.n. (INPA 48220); Thomas, W. et al. 5256. }\end{array}$ \\
\hline Iryanthera lancifolia Ducke & 5 & $\begin{array}{l}\text { Cid, C. A. et al. 6591, 6954, 7553; Loureiro, A. et al. s.n. (INPA 43848); Thomas, W. et } \\
\text { al. } 5309 .\end{array}$ \\
\hline Iryanthera macrophylla (Benth.) Warb. & 2 & Cid, C. A. et al. 67; Rodrigues, W. A. et al. 9342. \\
\hline Iryanthera paradoxa (Schw.) Warb. & 4 & $\begin{array}{l}\text { Amaral, I. L. et al. 1760; Cid, C. A. et al. 6665; Falcão, M. et al. 131; Monteiro, 0. P. et al. } \\
1362 .\end{array}$ \\
\hline Iryanthera paraensis Huber & 4 & Cid, C. A. et al. 81; Coelho, D. et al. 808; Prance, G. T. et al. 22660, 22662. \\
\hline Iryanthera polyneura Ducke & 2 & Cid, C. A. et al. 05; Steward, W. C. et al. P20410. \\
\hline Iryanthera sagotiana (Benth.) Warb. & 1 & Prance, G. T. et al. 22707. \\
\hline Iryanthera tricornis Ducke & 4 & Amaral, I. L. et al. 1720, 1735; Cid, C. A. et al. 8230; Silva, F. R. 133. \\
\hline Iryanthera ulei Warb. & 17 & $\begin{array}{l}\text { Amaral, I. L. et al. 1719, 1736; Berg, C. C. et al. P19526; Bisby, F. et al. P18058; Cid, C. } \\
\text { A. et al. 6634, 6692, 6878; Loureiro, A. et al. s.n. (INPA 48003); s.n. (INPA 48097), s.n. } \\
\text { (INPA 48124), s.n. (INPA 48196); Prance, G. T. et al. 22675, 22685, 22702, 24310A; } \\
\text { Rodrigues, W. A. et al. 9336, s.n. (INPA 48236). }\end{array}$ \\
\hline $\begin{array}{l}\text { Osteophloeum platyspermum (Spruce ex A. } \\
\text { DC.) Warb. }\end{array}$ & 5 & Cid, C. A. 7606; Cid, C. A. et al. 13, 8229; Silva, F. R. 82, 83. \\
\hline Virola caducifolia W. Rodr. & 1 & Thomas, W. et al. 5395. \\
\hline Virola calophylla (Spruce) Warb. var. calophylla & 14 & $\begin{array}{l}\text { Cid, C. A. et al. 33, 961, 6667, 7057, 8201, 11088; Freitas, C. A. A. et al. 241; Lima, } \\
\text { J. 581; Prance, G. T. et al. 24325; Steward, W. C. et al. 53, 56; Thomas, W. et al. 5251, } \\
\text { 5284, 5368. }\end{array}$ \\
\hline
\end{tabular}




\begin{tabular}{|c|c|c|}
\hline Famílias/Espécies & $\begin{array}{l}N^{0} \text { de } \\
\text { Ocorrência }\end{array}$ & Nome e $\mathrm{N}^{0}$ de Coletores \\
\hline $\begin{array}{l}\text { Virola calophylla var. calophylloidea (Mgf.) W. } \\
\text { Rodr. }\end{array}$ & 2 & Cid, C. A. et al. 08, 33A. \\
\hline Virola duckei A. C. Smith. & 3 & Coelho, D. et al. 803; Prance, G. T. et al. 24331; Rodrigues, W. A. et al. 10290. \\
\hline Virola elongata (Benth.) Warb. & 5 & $\begin{array}{l}\text { Cid, C. A. et al. 6905, 8253: Dionisia, F. et al. 173; Prance, G. T. et al. 23593; Steward, W. } \\
\text { C. et al. } 50 .\end{array}$ \\
\hline Virola mollissima (Poepp. ex A. DC.) Warb. & 5 & $\begin{array}{l}\text { Bisby, F. et al. P18120; Cid, C. A. et al. 8083; Monteiro, 0. P. et al. 1360; Silva, J. A. et al. } \\
\text { 821; Steward, W. C. et al. } 108 .\end{array}$ \\
\hline Virola multinervia Ducke & 6 & $\begin{array}{l}\text { Cid, C. A. et al. 8076; Monteiro, 0. P. et al. 1358, 1363; Thomas, W. et al. 5239, 5240; } \\
\text { Vieira, M. G. G. et al. } 1340 .\end{array}$ \\
\hline Virola pavonis (A. DC.) A. C. Smith. & 5 & Cid, C. A. et al. 6625, 7558; Prance, G. T. et al. 22699; Thomas, W. et al. 5295, 5308. \\
\hline Virola polyneura W. Rodr. & 1 & Silva, F. R. 142. \\
\hline Virola rugulosa (Spruce) Warb. & 1 & Monteiro, 0. P. et al. 1361. \\
\hline Virola sebifera Aubl. & 4 & Cid, C. A. et al. 6830, 8117; Prance, G. T. et al. 21633; Steward, W. C. et al. P19682. \\
\hline Virola venosa (Benth.) Warb. & 6 & $\begin{array}{l}\text { Amaral, I. L. et al. 1752, 1758; Monteiro, 0. P. et al. 1364, 1365; Thomas, W. et al. 5255, } \\
5270 .\end{array}$ \\
\hline \multicolumn{3}{|l|}{ Siparunaceae } \\
\hline Siparuna amazonica Mart. ex A. DC. & 1 & Rodrigues, W. A. et al. 9360. \\
\hline Siparuna cf. argyrochrysea Perk. & 1 & Prance, G. T. et al. 21641. \\
\hline Siparuna cristata (Poepp. \& Endl.) A. DC. & 6 & $\begin{array}{l}\text { Bisby, F. et al. P18056, P18062; Cid, C. A. et al. 6706, 86; Dionisia, F. et al. 205; Prance, } \\
\text { G. T. et al. } 24240 .\end{array}$ \\
\hline Siparuna cuspidata (Tul.) A. DC. & 2 & Cid, C. A. et al. 26; Silva, J. A. et al. 841. \\
\hline Siparuna decipiens (Tul.) A. DC. & 2 & Freitas, C. A. A. et al. 278; Thomas, W. et al. 5348. \\
\hline Siparuna guianensis Aubl. & 1 & Silva, M. F. et al. 1989. \\
\hline Siparuna lepidiflora Perk. & 2 & Prance, G. T. et al. 22668, 22687. \\
\hline Siparuna cf. mollicoma (Mart. ex Tul.) A. DC. & 1 & Amaral, I. L. et al. 1761. \\
\hline Siparuna sp. & 3 & Amaral, I. L. et al. 1763; Cid, C. A. et al. 6964; Freitas, C. A. A. et al. 175. \\
\hline \multicolumn{3}{|l|}{ Monimiaceae } \\
\hline Mollinedia ovata Ruiz \& Pav. & 1 & Silva, J. A. et al. 846. \\
\hline
\end{tabular}

\section{BIBLIOGRAFIA CITADA}

Belota Filho, W.L. 1994. As espécies de Iryanthera Warburg (Myristicaceae) da Reserva Florestal Ducke. Disssertação de Mestrado. INPA/FUA, Manaus, AM. 94pp.

De Candolle, A. 1860. Myristicaceae. In: Martius, K.F.P. von, Flora Brasiliensis, 5(1): 105-136.

Eletronorte/IBAMA. 1996. Reserva Biológica do Uatumã - Plano de Manejo - Fase 1. Documento de Informações Básicas. Manaus. $125 \mathrm{pp}$.

Gray Herbarium Index. 1968-1978. Havard University. G. K. Hall \& Co. Boston. vol. 1-10, supl. 1-2.

Greuter, W.; McNeil, J.; Barrie, F.R.; Burdet, H.M.; Demoulin, V.; Filgueiras, T.S.; Nikolson, D.H.; Silva, P.C.; Skog, J.E.; Trehane, P.; Turland, N.J.; Hawksworth, D.L. 2003. Código Internacional de Nomenclatura Botânica (Código de Saint Louis). Traduzido por C.E.M. Bicudo e J. Prado. Instituto de Botânica, São Paulo, SP. $162 \mathrm{pp}$.

Hooker, J.D.; Jackson, B.D. 1895-1974. Index Kewensis. Plantarum Phanerogamarum Nomina et synonima generum et specium. Oxford, Clarendron Press. vol. I-II, supl. I-XVIII.

Köppen, W.1948. Climatologia: con un estudio de los climas de la tierra. Fondo de Cultura Econômica. México. 479pp.
Le Cointe, P. 1947. Amazônia brasileira. III - Árvores e Plantas Úteis (indigenas e aclimatadas). $8^{\mathrm{a}}$ ed. São Paulo, Ed. Nacional. 506pp.

Loureiro, A.A.; Freitas, M.C.; Vasconcelos, F.J. 1989. Estudo Anatômico de 24 espécies do gênero Virola (Myristicaceae) da Amazônia. Acta Amazônica, 19(Único): 415-465.

Nava, D.B.; Monteiro, E.A.; Correia, M.C.; Araújo, M.R.; Sampaio, R.R.L.; Campos, G.S. 1998. Companhia de Pesquisa de Recursos Minerais/CPRM. Sócio-Economia do Município de Presidente Figueiredo, Amazonas. 63pp.

Nee, M. 1995. Flora Preliminar do Projeto Dinâmica Biológica de Fragmentos Florestais, New York Botanical Garden e INPA/ Smithsonian Projeto Dinâmica Biológica de Fragmentos Florestais. 264pp.

Ribeiro, J.E.L.S.; Hopkins, M.J.G.; Vicentini, A.; Sothers, C.A.; Costa, M.A.S.; Brito, J.M.; Souza, M.A.D.; Martins, L.H.P.; Lohmann, L.G.; Assunção, P.A.C.L.; Pereira, E.C.; Silva, C.F.; Mesquita, M.R.; Procópio, L.C. 1999. Flora da Reserva Ducke: Guia de identificação das plantas vasculares de uma floresta de terra firme na Amazônia Central. Manaus, INPA. 816pp. 
Rodrigues, W.A. 1980. Revisão Taxonômica das espécies de Virola Aublet (Myristicaceae) do Brasil. Tese de Doutorado. Universidade de Campinas / UNICAMP. vol. I e II.

Schodde, R. 1970. Two New Suprageneric Taxa in the Monimiaceae Alliance (Laurales). Taxon, 19(3): 324-325.
Stafleu, F.A. 1967. Taxonomic Literature. A selective guide to botanical publications with dates comentaries and Types. Utrech. Regnum Vegetabili, 52:556.

Tulasne, L.R. 1857. Monimiaceae. In: Martius, K.F.P. von, Flora Brasiliensis, 4(1): 289-328.

Recebido em 19/04/2006

Aceito em 06/02/2008 